\title{
Modeling and Design of a Humanoid Robot by Additive Manufacturing Process
}

\author{
J. Azeta*1, C. A. Bolu' ${ }^{2}$ F. A. Oyawale ${ }^{2}$ \\ 1, Ph.D., Students, Department of Mechanical Engineering, Covenant University, Ota, Ogun state, Nigeria. \\ ${ }^{2}$ Professor, Department of Mechanical Engineering, Covenant University, Ota, Ogun state, Nigeria.
}

\begin{abstract}
By the year 2030, it is estimated that 800 million workers all over the world could be replaced by robots and this is evident of a robotic revolution happening in healthcare worldwide. This study aims to model and design a humanoid robot with additive manufacturing process. Computer aided design (CAD) models of the selected concepts were developed in Fusion360 and imported into SolidWorks to optimise and improve the design. The first stage of the design focused on the development of the main body and wheelbase, while the second phase focused on the arms and manipulators. To meet the project requirement of a payload of $500 \mathrm{~g}$ we selected a tough and low density material with appropriate servo motor with a torque of $9.4 \mathrm{~kg} / \mathrm{cm}$ at each joint. The parts of the robot were produced by additive manufacturing process with an extrusion technique called fused deposition modelling (FDM) where a continuous filament of thermoplastic material, Polylactic acid (PLA) was fed from a coil via a moving heated extruder head and gradually deposit on the work. Overall, the robot proved that the proposed design is able to achieve the design requirements while mimicking basic human movements.
\end{abstract}

Keywords: Humanoid Robot, healthcare, Fused Deposition Modeling, Polylactic acid, Manipulator.

\section{INTRODUCTION}

The development of robots for use in a range of applications has become increasingly common over the years, particularly to replace repetitive tasks or in potentially dangerous situations (Hill \& Page, 2011; Lundeen et al., 2019; Shah \& Pandey, 2018). However, while the field of robotics is fast growing, the more specific area of hospital service robots for infectious disease treatment remains relatively underdeveloped (Azeta et al., 2018). Healthcare service robots are expected to play a crucial role in the development of a supportive tool to aid medical professionals in the treatment of infectious diseases which would increase the quality of life of both healthcare providers and patients (Mann et al., 2015; Ramoly et al., 2018; Yi et al., 2008). Advancement in robotic technology results in the integration of various sophisticated technologies such as mechanical engineering, electronics, control systems, and software which plays a significant role in automating the healthcare sector (Kumar et al., 2017). With the recent event of the unprecedented outbreak of corona virus, more interest has to be made towards the use of robot technology in healthcare.
Mobile humanoid robot platform can execute various services for humans in their everyday environments (cha et al., 2011). Wheeled mobile robots capable of operating safely and accurately in dynamic environments can have vast applications ranging from simple delivery tasks to advanced assembly operations (Costa et al., 2016).

\section{REVIEW}

Prototyping is a process of functional physical models and it is known as one of the most vital product development techniques. By creating scaled objects faults and flaws can easily be checked in the manufacturing process. In prototyping functional parts are checked for errors when they are connected or overloaded. CAD models are usually created in software's like Fusion360, AutoCAD, Solidworks, etc. Fabrication is usually done after virtual analysis of the object in analysing software's such as COMSOL, ANSYS, ABAQUS, SOLIDWORKS, etc. (Rajaguru et al., 2020).

The advancement of manufacturing technologies has led to the reduction of manufacturing time by lowering cost and lead time. Advanced design methodologies are used to leverage on creativity and design intent. The fuse deposition modelling (FDM) machines are the most popular additive manufacturing machines. In FDM heat chambers are used to liquefy polymer, which is then fed into the system as filament. The nozzle for extrusion is located on the $\mathrm{x}, \mathrm{y}$ and $\mathrm{z}$ axes commonly known as the Cartesian coordinate (Oosthuizen et al., 2013). In fused deposition modelling the filament is fed into the heating chamber where it is melted into a semisolid state and fed out the nozzle at a certain rate, depending on the relative rate of movement of the three axes, reading the g-code generated by the slicing software. The nozzle temperature is normally around $170{ }^{\circ} \mathrm{C}$ to $220{ }^{\circ} \mathrm{C}$ this temperature depends on the type of material that is used as a filament. The nozzle diameter is vital since the resolution of the print relies on the nozzle thickness and the rate of feed of the filament. Usually a nozzle has a diameter in about $0.35 \mathrm{~mm}$ to $0.5 \mathrm{~mm}$ (Oosthuizen et al., 2013). The advantage of AM is that it helps in accommodating highly complex design, reduce material wastage, restore damage parts, etc. (Rajaguru et al., 2020). The main disadvantage of using FDM technology is its manufacturing speed which is due to the inertia of the plotting heads. 


\section{METHOD}

The design consists of the body, the platform and the arms and end-effector. The body comprises the head, shoulder and torso support. The various parts are integrated as a whole.

\section{III.I Body Design}

The body is a vital part of the robot; it provides stability for the arms. It comprises of the head, shoulder, torso and waist for side turning movement. To meet the design requirement, the body was made of a lightweight Polylactic acid (PLA) material. The factors considered as basis of evaluation of the robot's parts when selecting the material are mass and stress. The mass of the system is dependent on the density of the selected material. Polylactic acid (PLA) is a thermoplastic polyester produced from renewable resources such as sugarcane, corn starch, etc. PLA is widely used in biomedical application and it is a tough plastic having a low density and high tensile strength which makes PLA the choice material for the robot body.

\section{III.I.II Design and manufacturing method}

In additive manufacturing technique materials are selectively deposited through an orifice, extruding materials in consecutive layers to produce items from 3D designs (CAD). With Fused Deposition Modeling (FDM), the production of a wide range of parts or functional components with complicated geometries, such as those acquired from a computer-aided design method is possible. FDM is ideal for concept models, functional prototypes, manufacturing aids and low volume end use parts. With the design specifications in mind, CAD models were designed with desired dimensions and tolerance. A tolerance of +0.02 was used for all the parts. With the CAD designs, adjustments were made to the dimensions and physical appearance of some parts. Fusion 360 was used to produce and visualize the design models. Materials were assigned, constraints were set, parts were assembled and the result was animated. Interferences were discovered in the assembly and were quickly adjusted to avoid wastage of time, material and energy after parts have been printed. Fig. 1. shows the various body parts such as the head, shoulder, shoulder support, torso, waist and waist support assembly. The design process led to a final design shown in Fig.2.

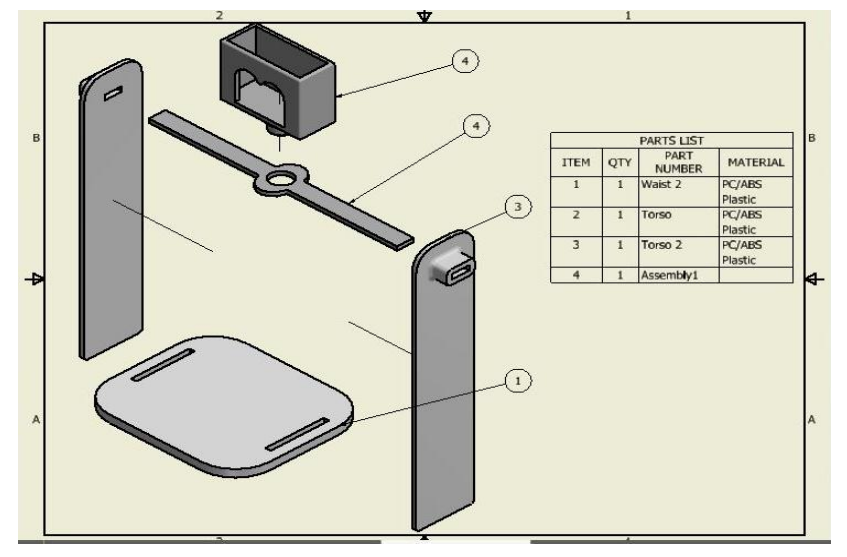

Figure. 1. Exploded view of the body parts

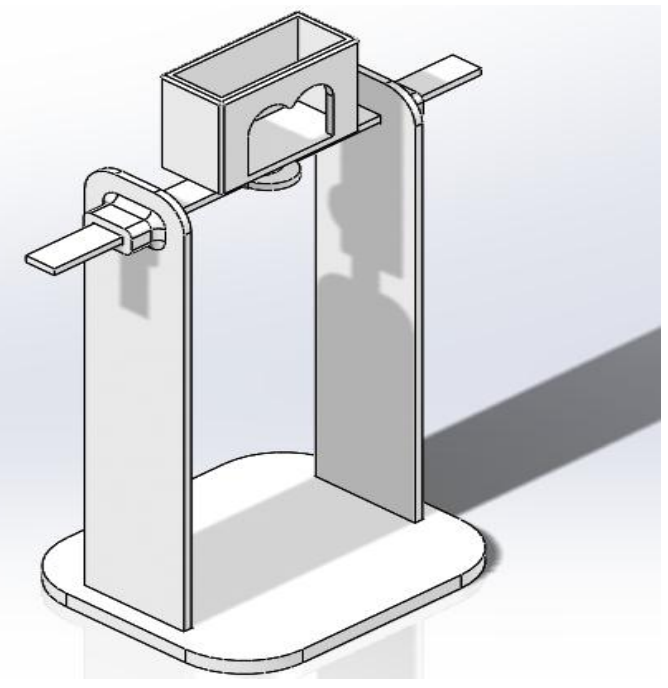

Figure. 2. Body assembly

\section{III.I.III Manufacture and assembly}

With Makerbot Replicator Z18, 3D printing of prototypes is made possible, which was used to produce the parts of the robots shown in Fig. 4(a-e), in the MakerBot Environment. But firstly, the CAD drawings were sliced, i.e. converted to Gcodes that the printer could understand. Slicing was also important because the slicing software is configured with all the features of the 3D printer to be used, so it understands the size of the printing platform and can display the model's position with respect to it. The model is then repositioned on the three axes (X, Y and $\mathrm{Z}$ ) until it is centered and lies precisely on the bed surface (not to be "suspended from air") and rotated if needed. The possibility to scale the dimensions of the model is also available.

In addition to this, slicing was necessary because there were a number of required additional parameters that needed to be provided, such as the thickness of the raft (which is a throwaway part of a print that mainly lays down a flat region on the print surface where the desired 3D model is produced), the height of the layers, infill density and supports. The steps to slicing and 3D printing are shown in Fig. 3.

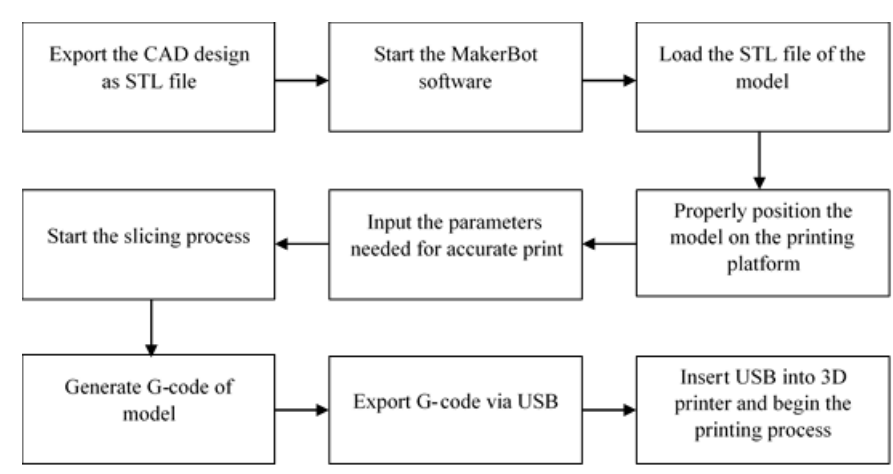

Figure. 3. Manufacturing value chain using 3D printing 


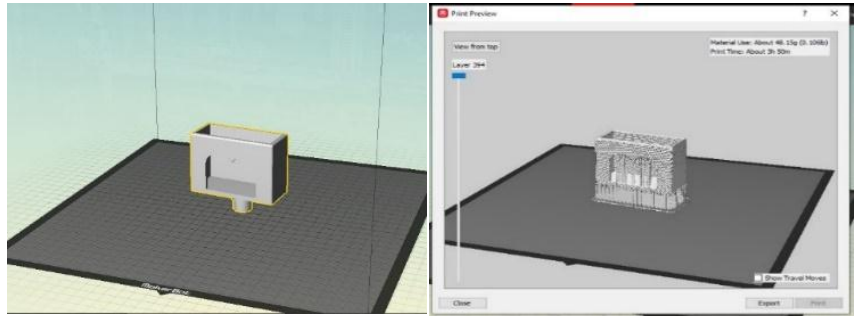

Figure. 4(a): The robot's head and the sliced view in the MakerBot environment

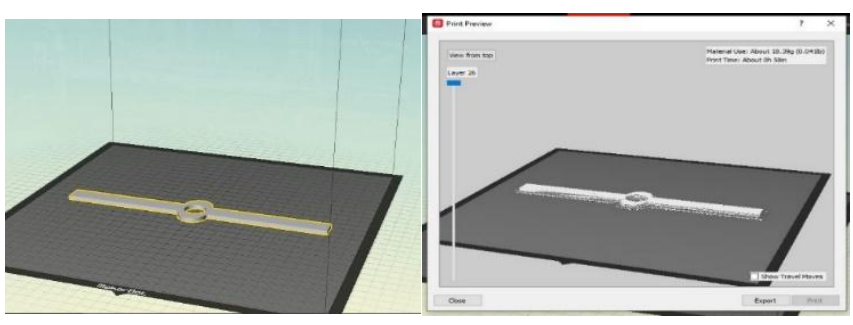

Figure. 4(b): The robot's shoulder and the sliced view in the MakerBot environment

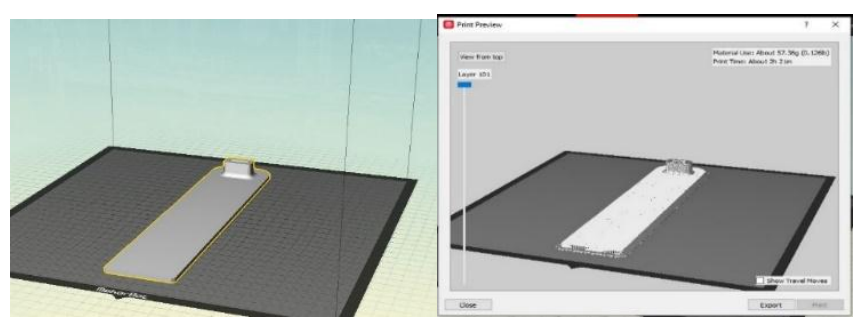

Figure. 4(c): The robot's torso and the sliced view in the MakerBot environment

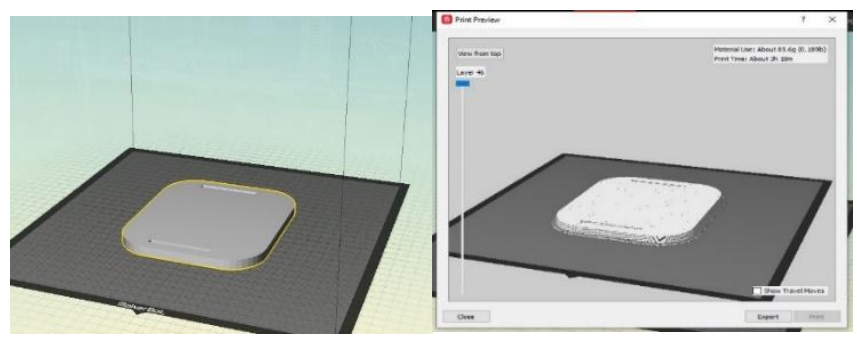

Figure. 4(d): The robot's waist and the sliced view in the MakerBot environment

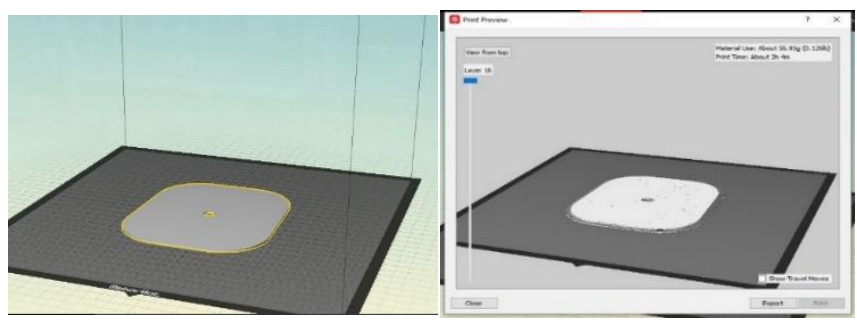

Figure 4(e): The waist support and the sliced view in the MakerBot environment

\section{III.II Wheelbase Design and Assembly}

The robots' mobile platform is a wheelbase with four drive wheels, two at the front and two at the rear which provides stability for the body and also a means of navigation. This type of platform is easy to control and is fit for indoor environment where there are no steps or big bumps. The requirements specify that the robot should have four wheels, and be able to drive on flat surfaces, which informed the design of the wheelbase shown in Fig 5.

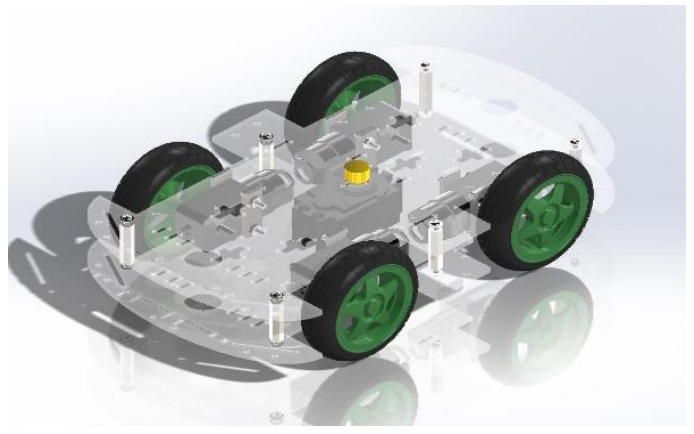

Figure. 5. Wheelbase Design

\section{III.III Arm and End-Effector}

The arm and end-effector includes two arms with links and injoint actuation. A manipulator was mounted at the end of the arm, to hold and move objects. The arms and end-effector provides needed motion. The design and development of the system requires a systematic process to make sure that the design was built to meet requirements. The arms and endeffectors were designed using the same additive manufacturing parts as used in the body. The joints are actuated by servo motors to achieve a total of 3 degrees of freedom (DOF); 1 DOF in the wrist, $1 \mathrm{DOF}$ in the elbow and $1 \mathrm{DOF}$ in the shoulder.

\section{III.III.I Arm torque requirements}

The main performance criteria for the arm was the torque required to perform the task, more specifically, the stall torque required for the actuator to maintain joint angles at maximum load condition. A payload of $500 \mathrm{~g}$ from project requirement with added weight of the arm itself gives the total payload for each arm. The Stall torque of the selected MG996R Servo motor $=9.4 \mathrm{~kg} / \mathrm{cm}$.

To determine the required torque $(\tau)$ at any lifting joint in the robot arm,

we have;

$\tau=F \times L$

Where,

$F=m g$

Therefore,

$\tau=m g \times L$ 
$\tau=$ torque needed to hold a mass at a given distance from a pivot.

$L=$ perpendicular length from pivot to force

$m g=$ weight of load

when we add the torque imposed by the robot arm itself, we have,

$\tau=(m g \times L)+(W \times L / 2)$
Simplified to;

$\tau=L(m g+W / 2)$

For uniform arm, the center of gravity can be considered to be at the middle of the arm

For worst case scenario, the arm is stretch at horizontal position. We then determine the torque required at each joint to select the appropriate motor as shown in Fig. 6.

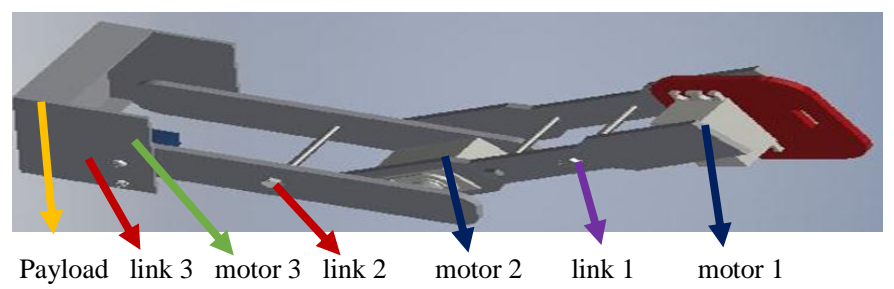

Figure. 6. Motor Selection for the Arm

The required torque for motors 3, 2, and 1 are calculated as follows:

$\tau_{3}=\left(m g \times L_{3}\right)+\left(W_{3} \times L_{3} / 2\right)$

$\tau_{3}=L_{3}\left(m g+W_{3} / 2\right)$

$\tau_{2}=\left[m g \times\left(L_{3}+L_{2}\right)\right]+\left[W_{3} \times\left(L_{2}+L_{3} / 2\right)\right]+\left(W_{m 3} \times L_{2}\right)+\left(W_{2} \times L_{2} / 2\right)$

$\tau_{2}=L_{3}+L_{2}\left(m g+W_{3} / 2\right)+L_{2}\left(W_{m 3}+W_{2} / 2\right)$

$\tau_{1}=\left[m g \times\left(L_{3}+L_{2}+L_{1}\right)\right]+\left[W_{3} \times\left(L_{1}+L_{2}+L_{3} / 2\right)\right]+\left[W_{m 3} \times\left(L_{1}+L_{2}\right)\right]+\left[W_{2} \times\left(L_{1}+L_{2} / 2\right)\right]+$

$\left(W_{\mathrm{m} 2} \times L_{1}\right)+\left(W_{1} \times L_{1} / 2\right)$

$\tau_{1}=L_{3}+L_{2}+L_{1}\left(m g+W_{3} / 2\right)+L_{2}+L_{1}\left(W_{m 3}+W_{2} / 2\right)+L_{1}\left(W_{m 2}+W_{1} / 2\right)$

Where,

$m g=$ weight on end -effector , that is the payload

$\operatorname{Link}_{3}, \operatorname{Link}_{2}, \operatorname{Link}_{1}=$ link length

Weight, ${ }_{3}$, Weight, ${ }_{2}$,Weight $=$ weight of link

Weight $t_{\mathrm{m} 3}$,Weight ${ }_{\mathrm{m} 2}$, Weight ${ }_{\mathrm{m} 1}=$ weight of motor

$m g=500 \mathrm{~g}$

Length of $\operatorname{Link}_{1}=14.0 \mathrm{~cm}$

Length of Link $=11.0 \mathrm{~cm}$

Length of Link $=5.7 \mathrm{~cm}$

Weight $=0.090 \mathrm{~kg}$

Weight $_{2}=0.043 \mathrm{~kg}$

Weight $=0.033 \mathrm{~kg}$

Weight motor $1=0.055 \mathrm{~kg}$

Weight $t_{\text {motor2 }}=0.055 \mathrm{~kg}$

Weight $t_{\text {motor8 }}=0.055 \mathrm{~kg}$ 
From equation 4, 5 and 6 we get the minimum torque requirement for the three motors on each robot arm with maximum load applied.

$\tau_{3}=5.7(0.5+0.033 / 2)$

$\tau_{3}=2.85+0.0165=2.87 \mathrm{~kg} / \mathrm{cm}$

$\tau_{2}=[5.7+11.0(0.5+0.033 / 2)]+[11.0(0.055+0.043 / 2)]$

$\tau_{2}=[8.35+0.28]+[0.61+0.24]=9.48 \mathrm{~kg} / \mathrm{cm}$

$\tau_{1}=[5.7+11.0+14.0(0.5+0.033 / 2)]+[11.0+14.0(0.055+0.043 / 2)]+[14.0(0.055+0.090 / 2)]$

$\tau_{1}=[15.35+0.51]+[1.38+0.54]+[0.77+0.63]=19.18 \mathrm{~kg} / \mathrm{cm}$

Using data from the overall robot requirements such as arm length and weight, the minimum torque requirement for motors 3,2 and 1 were obtained.

Considering the maximum load for each joint base on the selected motor.

From equation 4, we have;

$\tau_{3}=5.7(m g+0.033 / 2)$

$9.4=5.7(\mathrm{mg}+0.0165)$

$9.4=5.7 \mathrm{mg}+0.09405$

$5.7 \mathrm{mg}=9.4-0.09405$

$m g=1.633 \mathrm{~kg}=1633 \mathrm{~g}$

From equation 5, we have;

$\tau_{2}=5.7+11.0(\mathrm{mg}+0.033 / 2)+[11.0(0.055+0.043 / 2)]$

$9.4=16.7(\mathrm{mg}+0.017)+(0.61+0.24)$

$9.4=16.7 \mathrm{mg}+0.28+(0.85)$

$16.7 \mathrm{mg}=9.4-0.28-(0.85)$

$m g=0.495 \mathrm{~kg}=495 \mathrm{~g}$

From equation 6 , we have;

$\tau_{1}=[5.7+11.0+14.0(\mathrm{mg}+0.033 / 2)]+[11.0+14.0(0.055+0.043 / 2)]+[14.0(0.055+0.090 / 2)]$

$9.4=30.7(\mathrm{mg}+0.017)+1.38+0.54+0.77+0.63$

$9.4=30.7 \mathrm{mg}+0.52+1.92+1.4$

$30.7 m g=9.4-0.52-1.92-1.4$

$m g=0.181 \mathrm{~kg}=181 \mathrm{~g}$

The calculation for the torque requirements for the selected motors and the maximum load requirement at any lifting joint of the arm, in this case joints 3, 2, and 1 was carried out. Joints 3 gave a maximum payload of $1633 \mathrm{~g}$ which is safe to lift a load of $500 \mathrm{~g}$ and above. Based on the selected motors, joints 2 and 1 can lift a load of $495 \mathrm{~g}$ and $181 \mathrm{~g}$ respectively below the maximum payload of $500 \mathrm{~g}$. therefore joints 2 and 1 will stall. From the torque calculation it is required to use a motor with a torque higher than $9.48 \mathrm{~kg} / \mathrm{cm}$ for joint 2 and higher than $19.18 \mathrm{~kg} / \mathrm{cm}$ for joint 1 .

\section{III.III.II Design and analysis}

The CAD model was developed in Fusion360, and optimize to improve the design using Solidworks. Detailed drawings with critical dimensions and tolerances of the arm and end-effector were prepared with adequate information to manufacture. The assembly is shown in Fig. 7. Fused deposition modelling (FDM) and PLA were selected as the choice process and material for the arms and end-effector respectively. Motion studies were carried out to evaluate the function of the designs, the study 
confirms that no interference of parts occurred during operation. The motions include mimicking basic human movements with 1 degrees of freedom for each joint shown in Fig. 8.

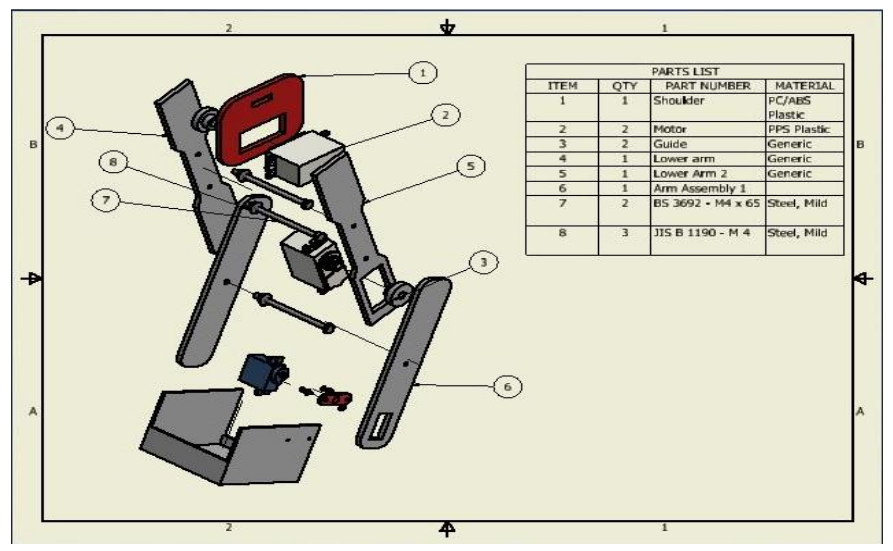

Figure 7. Exploded View of the Arm Assembly

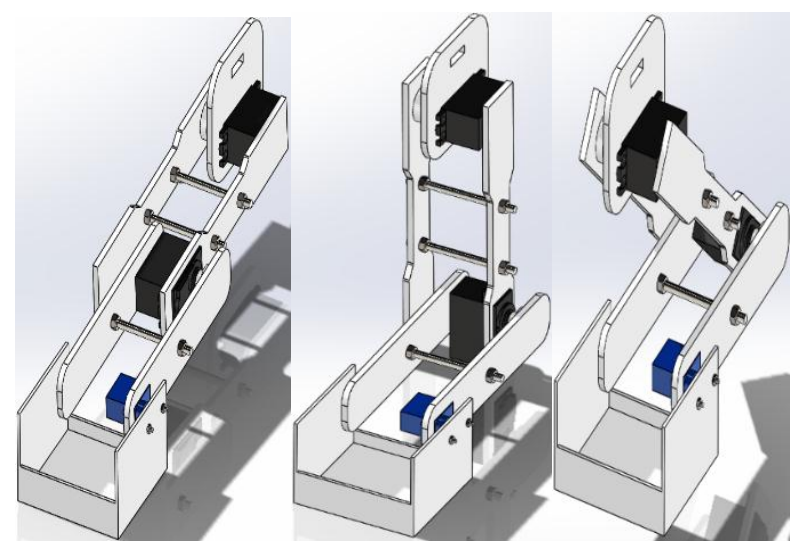

Figure 8. Arm Motion Analysis

\section{III.III.III Manufacture and assembly}

Fig. 9 shows the final assembly of the humanoid robot prototype. The 3D printed arm and end-effector were prepared in the same way as the body, employing FDM approach with PLA. The Makerbot Replicator Z18 was used to produce the arms and end-effector shown in Fig. 10.
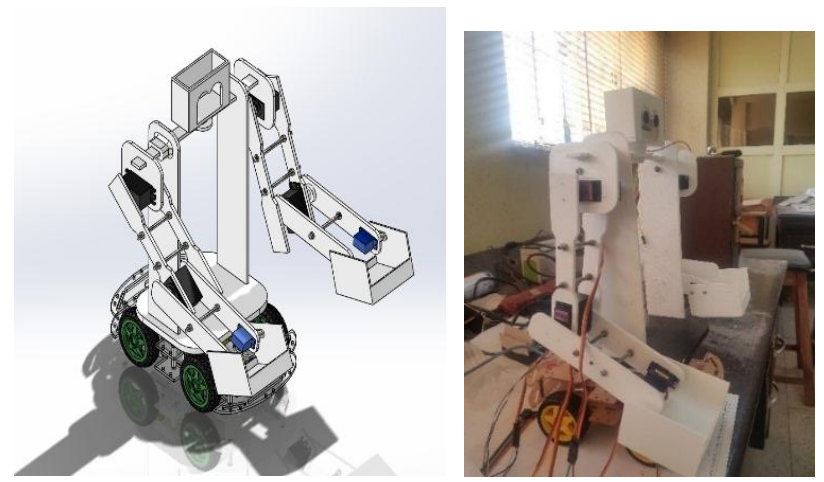

Figure. 9. Final Robot Assembly Figure. 10. Manufactured robot

\section{RESULT}

Stress study result of the critical stressed regions

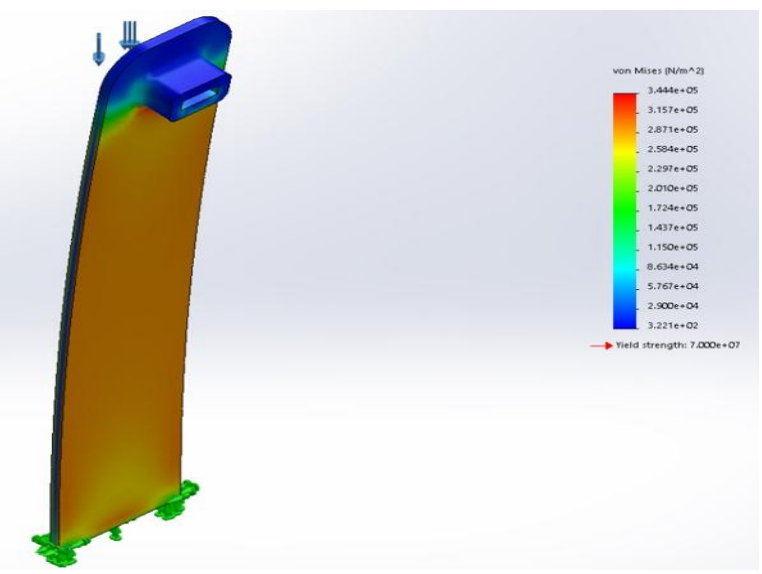

Figure. 11. Torso Stress Study Result

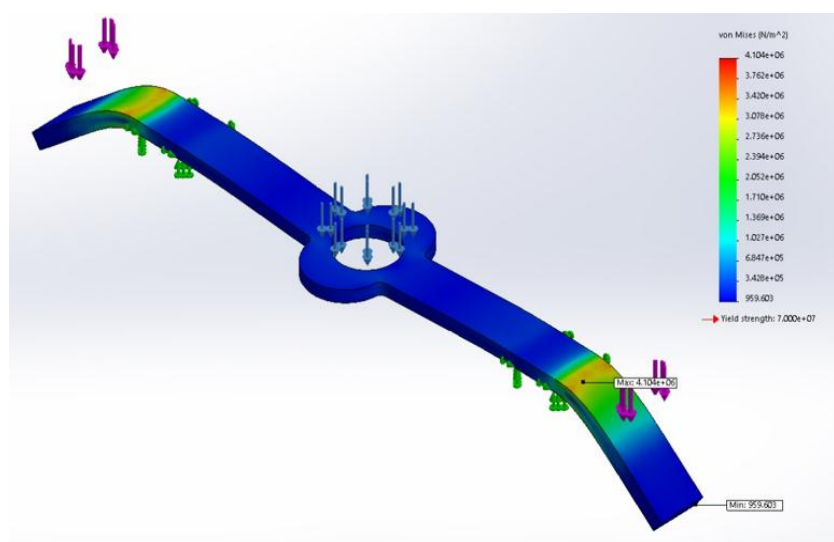

Figure. 12. Shoulder Stress Study Resul

\section{DISCUSSION}

The torso and the shoulder experience significant loads from the total weight of both arms and shoulder of the robot. The section of the torso that the load is applied was measured, and a load of $5.3499 \mathrm{~N}$ was applied on the torso. The material used in the study is Polylactic acid, with a yield strength of $59 \mathrm{MPa}$ and ultimate tensile strength of $70 \mathrm{MPa}$. Fig. 11 and 12 shows that the stress distribution is typical of bending and the maximum possible yield strength that can cause deformation of the part is $59 \mathrm{MPa}$. The applied load of $5.3499 \mathrm{~N}$ caused a maximum stress of $0.3444 \mathrm{MPa}$ and $4.104 \mathrm{MPa}$ which is far below the yield strength.

\section{CONCLUSION}

In this paper, a prototype humanoid robot with a payload of 500 $\mathrm{g}$ have been designed and the manipulation function is established by simulating the arm motion, which can be controlled remotely with wireless technology to support health personnel in delivery and other related tasks. The mobile platform provides stability for the robot and also a means of navigation. Finite element analysis was carried out on the 
critical stress region on the body such as the shoulder and torso support and the result shows that the stress distribution is typical of bending and the applied stress from the total weight of both arms on the shoulder and torso support is less than the yield strength of the material thereby preventing failure.

\section{REFERENCES}

[1] Azeta, J., Bolu, C., Abioye, A. A., \& Oyawale, F. A. (2018). A review on humanoid robotics in healthcare. MATEC Web of Conferences 153, 02004. https://doi.org/10.1051/matecconf/201815302004

[2] Oosthuizen, G. A., Hagedorn-Hansen, D., \& Gerhold, T. (2013). Evaluation of rapid product development technologies for production of prosthesis in developing communities.

[3] Costa, C. M., Sobreira, H. M., Sousa, A. J., \& Veiga, G. M. (2016). Robust 3/6 DoF self-localization system with selective map update for mobile robot platforms. Robotics and Autonomous Systems, 76, 113-140.

[4] Rajaguru, K., Karthikeyan, T., \& Vijayan, V. (2020). Additive manufacturing-State of art. Materials Today: Proceedings, 21, 628-633.

[5] Kumar, R., Jitoko, P., Kumar, S., Pillay, K., Prakash, P., Sagar, A., ... \& Mehta, U. (2017). Maze solving robot with automated obstacle avoidance. Procedia Computer Science, 105, 57-61.

[6] Ramoly, N., Bouzeghoub, A., \& Finance, B. (2018). A framework for service robots in smart home: an efficient solution for domestic healthcare. IRBM, 39(6), 413-420.

[7] Mann, J. A., MacDonald, B. A., Kuo, I. H., Li, X., \& Broadbent, E. (2015). People respond better to robots than computer tablets delivering healthcare instructions. Computers in Human Behavior, 43, 112-117.

[8] Yi, S., Moon, D., Yang, Y., \& Kim, K. (2008). Healthcare robot technology development. IFAC Proceedings Volumes, 41(2), 5318-5323.

[9] Lundeen, K. M., Kamat, V. R., Menassa, C. C., \& McGee, W. (2019). Autonomous motion planning and task execution in geometrically adaptive robotized construction work. Automation in Construction, 100, 24-45.

[10]Hill, V., \& Page, S. (2011). Poster 91 The Efficacy of Using a Combined Regimen of Portable Robotics and a Repetitive Task Specific Practice to Increase Motor Function in the Upper Arm. Archives of Physical Medicine and Rehabilitation, 92(10), 1718.

[11] Shah, R., \& Pandey, A. B. (2018). Concept for automated sorting robotic arm. Procedia Manufacturing, 20, 400-405.

[12] Cha, Y. S., Kim, K., Lee, J. Y., Lee, J., Choi, M., Jeong, M. H., ... \& Oh, S. R. (2011). MAHRU-M: A mobile humanoid robot platform based on a dual-network control system and coordinated task execution. Robotics and Autonomous Systems, 59(6), 354-366. 